\title{
RELATION BETWEEN GROWTH AND REGULARITY OF SOLUTIONS OF HYPOELLIPTIC EQUATIONS
}

\author{
M. SHAFII-MOUSAVI AND Z. ZIELEZNY \\ (Communicated by Walter D. Littman)
}

\begin{abstract}
For a class of linear partial differential equations with variable coefficients, it is shown that the Gevrey regularity of solutions depends on their growth at infinity.
\end{abstract}

Let $P(D)$ be a partial differential operator with constant coefficients. If $P(D)$ is hypoelliptic, then all distributions $u$ in $R^{n}$, solutions of the equation

$$
P(D) u=0 \text {, }
$$

are $C^{\infty}$-functions which belong to a Gevrey class $\Gamma^{d}\left(R^{n}\right)$, where $d=\left(d_{1}, \ldots, d_{n}\right)$ and $d_{j} \geq 1, j=1, \ldots, n$. This means that for every compact set $K \subset R^{n}$ there is a constant $C>0$ such that

$$
\left|D^{\alpha} u(x)\right| \leq C^{|\alpha|+1} \alpha_{1}^{\alpha_{1} d_{1}} \alpha_{2}^{\alpha_{2} d_{2}} \cdots \alpha_{n}^{\alpha_{n} d_{n}}, \quad x \in K
$$

for every multi-index $\alpha$.

V. V. Grusin [2] has shown that, for a given solution $u$ of equation (1), the Gevrey class $\Gamma^{d}\left(R^{n}\right)$ depends not only on the differential operator $P(D)$ but also on the growth of $u$ at infinity. In fact, the numbers $d_{j}, j=1, \ldots, n$, can be lowered and condition (2) can be replaced by a global condition in $R^{n}$, if one considers solutions of finite exponential order of growth in $R^{n}$.

The aim of this paper is to extend Grusin's investigations to a class of partial differential operators with variable coefficients.

1. The case of operators with constant coefficients. We recall briefly some of the results obtained in [2].

Let $P(D)$ be a differential operator with constant coefficients and $P(\xi)$ the corresponding polynomial. We denote by $\mathscr{N}_{k}$ the set of all $\zeta=\left(\varsigma_{1}, \ldots, \varsigma_{n}\right) \in \mathbf{C}^{n}$ such that $P(\varsigma)=0$ and $\operatorname{Im} \zeta_{j}=0$ for $j \neq k$. If $P(D)$ is hypoelliptic, then there are rational numbers $d_{j}^{k}>0$ and constants $C_{j}^{k}>0, j, k=1, \ldots, n$, with the following properties:

$\left(\mathrm{h}_{1}\right)$ If $\varsigma=\xi+i \eta$, where $\xi=\left(\xi_{1}, \ldots, \xi_{n}\right)$ and $\eta=\left(\eta_{1}, \ldots, \eta_{n}\right)$ are in $R^{n}$, then

$$
\left|\xi_{j}\right| \leq C_{j}^{k}(1+|\eta|)^{d_{j}^{k}}=C_{j}^{k}\left(1+\left|\eta_{k}\right|\right)^{d_{j}^{k}} .
$$

$\left(\mathrm{h}_{2}\right) d_{j}^{k}$ are the smallest numbers for which the inequalities (3) are valid with some constants $C_{j}^{k}$.

Received by the editors March 2, 1988.

1980 Mathematics Subject Classification (1985 Revision). Primary 35B65, 35J30.

The first author was partially supported by a Grant-in-aid of Research from Indiana University at South Bend. The contents of this paper have been presented at the Atlanta Meeting of the American Mathematical Society on January 6, 1988. 
We call the numbers $d_{j}^{k}$ the exponents of hypoellipticity of the operator $P(D)$. We note that $d_{j}^{k}$ corresponds to $1 / \gamma_{j}^{k}$ in [2].

It is well known that every differential operator $P(D)$ with constant coefficients has a tempered fundamental solution, i.e. there exists a tempered distribution $E$ such that

$$
P(D) E=\delta
$$

where $\delta$ is the Dirac measure. For a hypoelliptic operator $P(D)$, every tempered fundamental solution $E$ has the following properties (see [2, Theorem 3.1]):

$\left(\mathrm{e}_{1}\right)$ If $d_{j}^{k}, k, j=1, \ldots, n$, are exponents of hypoellipticity of $P(D)$ and $d_{j}=$ $\max _{1 \leq k \leq n} d_{j}^{k} \geq 1$, then $E \in \Gamma^{d}\left(R^{n} \backslash\{0\}\right)$, where $d=\left(d_{1}, \ldots, d_{n}\right)$.

$\left(\mathrm{e}_{2}\right)$ There exists an integer $l \geq 0$ such that

$$
D^{\alpha} E(x)=O\left((1+|x|)^{l}\right) \quad \text { as }|x| \rightarrow \infty,
$$

for every multi-index $\alpha$.

In particular, the property $\left(e_{1}\right)$ implies that every distribution $u$, solution of equation (1) in $R^{n}$, belongs to $\Gamma^{d}\left(R^{n}\right)$ (see [4, Proposition 7.2, p. 413]).

We now state a version of the theorem of Grusin [2, Theorem 4.1]) which establishes the relation between growth and regularity of solutions of equation (1).

THEOREM 1. Let $P(D)$ be a hypoelliptic differential operator and $d_{j}^{k}, j, k=$ $1, \ldots, n$, its exponents of hypoellipticity. Furthermore, let $u$ be a solution of equation (1) which satisfies the growth condition

$$
|u(x)| \leq A \exp \left(a \sum_{k=1}^{n}\left|x_{k}\right|^{p_{k}}\right), \quad x \in R^{n},
$$

where $A$ and $a$ are positive constants and $p_{k}>1, k=1, \ldots, n$. Then there exist constants $C>0$ and $c \geq a$ such that

$$
\left|\frac{\partial^{m} u(x)}{\partial x_{j}^{m}}\right| \leq A C^{m}\left(\sum_{k=1}^{n} m^{m d_{j}^{k} / q_{k}}\right) \exp \left(c \sum_{k=1}^{n}\left|x_{k}\right|^{p_{k}}\right), \quad x \in R^{n},
$$

where $m=1,2, \ldots$, and $1 / p_{k}+1 / q_{k}=1$.

REMARK. Since $p_{k}>1$, clearly $d_{j}^{k} / q_{k}<d_{j}^{k}, j, k=1, \ldots, n$, which shows that the regularity is improved due to the restriction of the growth of $u$.

2. The case of operators with variable coefficients. We consider a differential operator of the form

$$
P(x, D)=P_{0}(D)+\sum_{\nu=1}^{r} a_{\nu}(x) P_{\nu}(D)
$$

where $P_{\nu}(D)$ are operators with constant coefficients and the functions $a_{\nu}$ satisfy certain regularity and growth conditions. Specifically, we make the following assumptions on the operators $P_{\nu}(D)$ :

$\left(c_{1}\right)$ The operator $P_{0}(D)$ is hypoelliptic. We denote by $d_{j}^{k}, j, k=1, \ldots, n$, its exponents of hypoellipticity and assume that $d_{j}^{k}>1$. 
$\left(c_{2}\right)$ For $j=1, \ldots, n$ and $\nu=1, \ldots, r$,

$$
\int_{R^{n}} \frac{\widetilde{\xi_{j} P_{\nu}}(\xi)}{\tilde{P}_{0}(\xi)} d \xi<\infty
$$

where $\tilde{P}(\xi)=\left(\sum_{\alpha}\left|P^{(\alpha)}(\xi)\right|^{2}\right)^{1 / 2}$ and $P^{(\alpha)}(\xi)=D^{\alpha} P(\xi)$.

Note that, because of $\left(c_{1}\right), P_{0}(D)$ satisfies the conditions imposed on $P(D)$ in Theorem 1. Also, condition ( $\left.c_{2}\right)$ implies that, for any $x_{0} \in R^{n}, P\left(x_{0}, D\right)$ and $P_{0}(D)$ are equally strong. Hence $P\left(x_{0}, D\right)$ is hypoelliptic for every $x_{0} \in R^{n}$, by $\left(c_{1}\right)$ and Theorem 4.1.6 in [3]. Moreover, if $a_{\nu}, \nu=1, \ldots, r$, are $C^{\infty}$-functions then every distribution $u$ in $R^{n}$, solution of the equation

$$
P(x, D) u=0,
$$

is a $C^{\infty}$-function, by Theorem 7.4.1 in [3]. We wish to study solutions of equation (10) which satisfy the growth condition (6).

We make the following assumption on the functions $a_{\nu}$ :

$\left(c_{3}\right)$ Let $\rho_{j}=\min _{1 \leq k \leq n} d_{j}^{k} / q_{k}, j=1, \ldots, n$, and let $c$ be the constant in (7), when Theorem 1 is applied to the operator $P_{0}(D)$. Then there exist constants $B>0$ and $b>c$ such that

$$
\left|D^{\alpha} a_{\nu}(x)\right| \leq B^{|\alpha|+1} \alpha_{1}^{\alpha_{1} \rho_{1}} \cdots \alpha_{n}^{\alpha_{n} \rho_{n}} \exp \left(-b \sum_{k=1}^{n}\left|x_{k}\right|^{p_{k}}\right), \quad x \in R^{n}
$$

for all $\nu=1, \ldots, r$, and all multi-indices $\alpha$.

REMARK. Since $d_{j}^{k}<1$ and $1 / p_{k}+1 / q_{k}=1$, we have $1 / p_{k}+d_{j}^{k} / q_{k}>1, j, k=$ $1, \ldots, n$, and therefore the family of functions satisfying condition $\left(c_{3}\right)$ is not trivial, i.e. it contains functions that are not identically zero (see [1, Chapter IV, §8]).

Our main result is the following theorem.

THEOREM 2. Let $P(x, D)$ be a differential operator of the form (8), where the operators $P_{0}(D)$ and $P_{\nu}(D)$ satisfy conditions $\left(c_{1}\right)$ and $\left(c_{2}\right)$, and the functions $a_{\nu}$ satisfy condition $\left(c_{3}\right)$. If $u$ is a solution of equation (10) which satisfies the growth condition (6), then there are positive constants $A_{1}, C_{1}$ and $c_{1}>a$ such that

$$
\left|\frac{\partial^{m} u(x)}{\partial x_{j}^{m}}\right| \leq A_{1} C_{1}^{m}\left(\sum_{k=1}^{n} m^{m\left(1+d_{j}^{k} / q_{k}\right)}\right) \exp \left(c_{1} \sum_{k=1}^{n}\left|x_{k}\right|^{p_{k}}\right), \quad x \in R^{n}
$$

where $m=1,2, \ldots$, and $1 / p_{k}+1 / q_{k}=1$.

We first prove two lemmas. In the first lemma, $\mathscr{S}$ denotes Schwartz's space of rapidly decreasing $C^{\infty}$-functions.

LEMMA 1. Let $P(D)$ and $Q(D)$ be differential operators with constant coefficients and suppose that $P(D)$ is hypoelliptic. If

$$
\int_{R^{n}} \frac{\tilde{Q}(\xi)}{\tilde{P}(\xi)} d \xi<\infty
$$

and if $E$ is a fundamental solution for $P(D)$, then there are constants $M$ and $N$ such that

$$
|(Q(D) E * \phi)(x)| \leq M(1+|x|)^{N} \int_{R^{n}}(1+|y|)^{N}|\phi(y)| d y, \quad x \in R^{n},
$$

where "*" denotes the convolution and $\phi \in \mathscr{S}$. 
PROOF. Since $P(D)$ is hypoelliptic, there are positive constants $a, c$ and $C$ such that

$$
|\xi|^{c} \leq C|P(\xi)| \text { for } \xi \in G_{a}=\left\{\eta \in R^{n} ;|\eta| \geq a\right\} .
$$

Consider the function

$$
\Phi(\xi)= \begin{cases}\frac{1}{P(-\xi)} & \text { for } \xi \in G_{a} \\ 0 & \text { otherwise. }\end{cases}
$$

If $\hat{E}$ is the Fourier transform of $E$, then $P \hat{E}=1$. In particular, $\hat{E}(\xi)=1 / P(\xi)$ for $\xi \in G_{a}$. It follows that $H=(\hat{E})^{\vee}-\Phi$ is a distribution with compact support and we have

$$
E=\hat{H}+\hat{\Phi}
$$

$(\hat{E})^{\sim}$ is defined by $(\hat{E})^{\sim}(\phi)=\hat{E}(\check{\phi})$, where $\check{\phi}(\xi)=\phi(-\xi)$.

By the Paley-Wiener-Schwartz theorem, $\hat{H}$ is an entire function of exponential type and there are positive constants $M_{1}$ and $N$ such that

$$
|Q(D) \hat{H}(x)| \leq M_{1}(1+|x|)^{N}, \quad x \in R^{n} .
$$

We note that $N$ does not depend on the operator $Q(D)$. It follows that, for any $\phi \in \mathscr{S}$, we have

$$
\begin{aligned}
|(Q(D) \hat{H} * \phi)(x)| & \leq M_{1} \int_{R^{n}}(1+|x-y|)^{N}|\phi(y)| d y \\
& \leq M_{1}(1+|x|)^{N} \int_{R^{n}}(1+|y|)^{N}|\phi(y)| d y, \quad x \in R^{n} .
\end{aligned}
$$

Also,

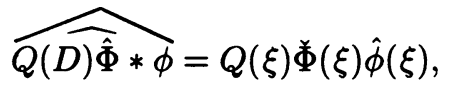

so that

$$
(Q(D) \hat{\Phi} * \phi)(x)=\frac{1}{(2 \pi)^{n}} \int_{R^{n}} e^{i\langle x, \xi\rangle} Q(\xi) \check{\Phi}(\xi) \hat{\phi}(\xi) d \xi
$$

Hence

$$
|(Q(D) \hat{\Phi} * \phi)(x)| \leq \frac{1}{(2 \pi)^{n}} \int_{G_{a}}\left|\frac{Q(\xi)}{P(\xi)}\right||\hat{\phi}(\xi)| d \xi, \quad x \in R^{n}
$$

Using again the hypoellipticity of $P(D)$, we can find a constant $C^{\prime}>0$ such that

$$
\tilde{P}(\xi) \leq C^{\prime}|P(\xi)|, \quad \xi \in G_{a} .
$$

Consequently,

$$
\begin{aligned}
|(Q(D) \hat{\Phi} * \phi)(x)| & \leq \frac{C^{\prime}}{(2 \pi)^{n}} \sup _{\xi \in R^{n}}|\hat{\phi}(\xi)| \int_{G_{a}} \frac{|Q(\xi)|}{\tilde{P}(\xi)} d \xi \\
& \leq M_{2} \int_{R^{n}}|\phi(y)| d y, \quad x \in R^{n}
\end{aligned}
$$

where

$$
M_{2}=\frac{C^{\prime}}{(2 \pi)^{n}} \int_{R^{n}} \frac{\tilde{Q}(\xi)}{\tilde{P}(\xi)} d \xi
$$

If $M=M_{1}+M_{2}$, we obtain from (15), (16) and (17) the estimate (14). 
COROLlaRY. If $P(D), Q(D)$ and $E$ are as in Lemma 1 and

$$
\int_{R^{n}} \frac{\widetilde{\xi_{j} Q}(\xi)}{\tilde{P}(\xi)} d \xi<\infty
$$

for some $j$, then

$$
\left|\left(Q(D) E * \frac{\partial \phi}{\partial x_{j}}\right)(x)\right| \leq M(1+|x|)^{N} \int_{R^{n}}(1+|y|)^{N}|\phi(y)| d y, \quad x \in R^{n} .
$$

For the proof it suffices to apply Lemma 1 to the operator $D_{j} Q(D)$, where $D_{j}=\partial / \partial x_{j}$.

REMARK. Lemma 1 remains valid, if we assume that $\phi$ is a continuous function rapidly decreasing at infinity, i.e. that $(1+|x|)^{k} \phi(x)$ is bounded in $R^{n}$, for every $k$.

LEMMA 2. Let $P(x, D)$ be a differential operator of the form $(8)$, where $P_{0}(D)$, $P_{\nu}(D)$ and $a_{\nu}, \nu=1, \ldots, r$, satisfy conditions $\left(\mathrm{c}_{1}\right),\left(\mathrm{c}_{2}\right)$ and $\left(\mathrm{c}_{3}\right)$, and let $E_{0}$ be a tempered fundamental solution for $P_{0}(D)$. Then there are operators $Q_{\nu}(D)$ and functions $b_{\nu}, \nu=1, \ldots, s$, with the following property. If $u$ is a solution of the equation (10) which satisfies condition (6), then

$$
v=u+\sum_{\nu=1}^{s} Q_{\nu}(D) E_{0} *\left(b_{\nu} u\right)
$$

is a solution of the equation

$$
P_{0}(D) v=0 \text {. }
$$

Each polynomial $Q_{\nu}(\xi)$ is a derivative of some order of a polynomial $P_{\mu}(\xi), \mu \geq 1$, and each function $b_{\nu}(\xi)$ is proportional to a derivative of some order of a function $a_{\mu}(\xi)$.

PROOF. The polynomials $Q_{\nu}(\xi)$ and the functions $b_{\nu}(\xi)$ obviously satisfy the conditions (9) and (11), respectively. In particular, the products $b_{\nu} u$ decrease at infinity faster than any power of $|\xi|^{-1}$. Therefore the convolutions in (19) are well defined.

By assumption

$$
P_{0}(D) u+\sum_{\nu=1}^{r} a_{\nu} P_{\nu}(D) u=P(x, D) u=0 .
$$

To each term $a_{\nu} P_{\nu}(D) u$ we now apply the generalized Leibniz formula

$$
a_{\nu} P_{\nu}(D) u=\sum_{\alpha} \frac{(-1)^{|\alpha|}}{\alpha !} P_{\nu}^{(\alpha)}(D)\left(u D^{\alpha} a_{\nu}\right)
$$

In this way we obtain the equation

$$
P_{0}(D) u+\sum_{\nu=1}^{s} Q_{\nu}(D)\left(b_{\nu} u\right)=0
$$

Since $E_{0}$ is a fundamental solution for $P_{0}(D)$, we have

$$
Q_{\nu}(D)\left(b_{\nu} u\right)=P_{0}(D)\left[Q_{\nu}(D) E_{0} *\left(b_{\nu} u\right)\right]
$$


for each $\nu=1, \ldots, s$. Hence

$$
P_{0}(D)\left[u+\sum_{\nu=1}^{s} Q_{\nu}(D) E_{0} *\left(b_{\nu} u\right)\right]=0
$$

which proves the lemma.

PROOF OF THEOREM 2. If $u$ satisfies condition (6), then $v$ satisfies the same condition with another constant $A_{0} \geq A$. Since, by Lemma $2, v$ is a solution of equation (20), we may apply Theorem 1 to conclude that

$$
u=-\sum_{\nu=1}^{s} Q_{\nu}(D) E_{0} *\left(b_{\nu} u\right)+f
$$

where $Q_{\nu}(D)$ and $b_{\nu}$ are as in Lemma 2 and $f$ is a $C^{\infty}$-function which satisfies the estimates (7). In particular, condition (18) is valid for $j=1, \ldots, n$, with $Q(\xi)$ replaced with $Q_{\nu}(\xi), \nu=1, \ldots, s$, and the functions $b_{\nu}$ satisfy condition (11) with the constants $B$ and $b$. Thus, in view of the corollary from Lemma 1 , we have

$$
\begin{aligned}
\left|\frac{\partial u(x)}{\partial x_{j}}\right| & \leq M \sum_{\nu=1}^{s}(1+|x|)^{N} \int_{R^{n}}(1+|y|)^{N}\left|b_{\nu}(y) u(y)\right| d y+\left|\frac{\partial f(x)}{\partial x_{j}}\right| \\
& \leq A_{0} C_{1}(1+\lambda s M) \exp \left(c \sum_{k=1}^{n}\left|x_{k}\right|^{p_{k}}\right), \quad x \in R^{n}
\end{aligned}
$$

where $C_{1}=\max \{B, C\}$ and

$$
\begin{aligned}
\lambda=\sup _{x \in R^{n}}\left[(1+|x|)^{N} \exp \left(-c \sum_{k=1}^{n}\left|x_{k}\right|^{p_{k}}\right)\right] \\
\cdot \int_{R^{n}}(1+|y|)^{N} \exp \left[-(b-c) \sum_{k=1}^{n}\left|x_{k}\right|^{p_{k}}\right] d y .
\end{aligned}
$$

Suppose now that

$$
\left|\frac{\partial^{l} u(x)}{\partial x_{j}^{l}}\right| \leq A_{0} C_{1}^{l}(l+\lambda s M)^{l}\left(\sum_{k=1}^{n} l^{l d_{j}^{k} / q_{k}}\right) \exp \left(c \sum_{k=1}^{n}\left|x_{k}\right|^{p_{k}}\right), \quad x \in R^{n}
$$

for $l=1, \ldots, m$. Then

$$
\begin{aligned}
& \left|\frac{\partial^{m+1} u(x)}{\partial x_{j}^{m+1}}\right| \leq M \sum_{\nu=1}^{s}(1+|x|)^{N} \int_{R^{n}}(1+|y|)^{N}\left|\frac{\partial^{m}\left[b_{\nu}(y) u(y)\right]}{\partial y_{j}^{m}}\right| d y+\left|\frac{\partial^{m+1} f(x)}{\partial x_{j}^{m+1}}\right| \\
& \leq A_{0} C_{1}^{m+1}\left\{\lambda s M \sum_{\nu=0}^{m}\left(\begin{array}{c}
m \\
l
\end{array}\right) l^{l \rho_{j}}(l+\lambda s M)^{m-l}\left(\sum_{k=1}^{n}(m-l)^{(m-l) d_{j}^{k} / q_{k}}\right)\right. \\
& \left.\quad+\left(\sum_{k=1}^{n}(m+1)^{(m+1) d_{j}^{k} / q_{k}}\right)\right\} \\
& \quad \exp \left(c \sum_{k=1}^{n}\left|x_{k}\right|^{p_{k}}\right), \quad x \in R^{n} .
\end{aligned}
$$


Since, by definition, $\rho_{j}=\min _{1 \leq k \leq n} d_{j}^{k} / q_{k}$, we have

$$
l^{l \rho_{j}}(m-l)^{(m-l) d_{j}^{k} / q_{k}} \leq l^{l d_{j}^{k} / q_{k}}(m-l)^{(m-l) d_{j}^{k} / q_{k}} \leq m^{m d_{j}^{k} / q_{k}},
$$

for each $k=1, \ldots, n$, and so

$$
l^{l \rho_{j}} \sum_{k=1}^{n}(m-l)^{(m-l) d_{j}^{k} / q_{k}} \leq \sum_{k=1}^{n} m^{m d_{j}^{k} / q_{k}} .
$$

Also,

$$
\begin{aligned}
\sum_{l=0}^{m}\left(\begin{array}{c}
m \\
l
\end{array}\right)(l+\lambda s M)^{m-l} & \leq \sum_{l=0}^{m}\left(\begin{array}{c}
m \\
l
\end{array}\right)[1+(l+\lambda s m)]^{m-l} \\
& \leq \sum_{l=0}^{m}\left(\begin{array}{c}
m \\
l
\end{array}\right)[1+(m+\lambda s M)]^{m-l}=(m+1+\lambda s M)^{m}
\end{aligned}
$$

Hence

$$
\begin{aligned}
\lambda s M & \sum_{l=0}^{m}\left(\begin{array}{c}
m \\
l
\end{array}\right)(l+\lambda s M)^{m-l}+1 \\
& \leq \lambda s M(m+1+\lambda s M)^{m}+1 \leq(m+1+\lambda s M)^{m+1} .
\end{aligned}
$$

It therefore follows from (21) that

$$
\begin{gathered}
\left|\frac{\partial^{m+1} u(x)}{\partial x_{j}^{m+1}}\right| \leq A_{0} C_{1}^{m+1}(m+1+\lambda s M)^{m+1}\left(\sum_{k=1}^{n}(m+1)^{(m+1) d_{j}^{k} / q_{k}}\right) \\
\cdot \exp \left(c \sum_{k=1}^{n}\left|x_{k}\right|^{p_{k}}\right), \quad x \in R^{n} .
\end{gathered}
$$

This proves, by induction, that for any $j=1, \ldots, n$, and $m=1,2, \ldots$,

$$
\begin{aligned}
\left|\frac{\partial^{m} u(x)}{\partial x_{j}^{m}}\right| & \leq A_{0} C_{1}^{m}(m+\lambda s M)^{m}\left(\sum_{k=1}^{n} m^{m d_{j}^{k} / q_{k}}\right) \exp \left(c \sum_{k=1}^{n}\left|x_{k}\right|^{p_{k}}\right) \\
& \leq A_{0} e^{\lambda s M} C_{1}^{m}\left(\sum_{k=1}^{n} m^{m\left(1+d_{j}^{k} / q_{k}\right)}\right) \exp \left(c \sum_{k=1}^{n}\left|x_{k}\right|^{p_{k}}\right), \quad x \in R^{n} .
\end{aligned}
$$

The theorem is thus established with the constants

$$
A_{1}=A_{0} e^{\lambda s M}, \quad C_{1} \text { and } \quad c_{1}=c .
$$

REMARK. If $1<\max _{1 \leq k \leq n} d_{j}^{k} / p_{k}$, then $1+\max _{1 \leq k \leq n} d_{j}^{k} / q_{k}<\max _{1 \leq k \leq n} d_{j}^{k}$, i.e. the regularity of the solution $u$ in the $j$ th variable is improved due to the growth condition (6). 


\section{REFERENCES}

1. I. M. Gelfand and G. E. Silov, Generalized functions, vol. 2, Academic Press, New York, 1968.

2. V. V. Grusin, Connection between local and global properties of solutions of hypoelliptic equations with constant coefficients, Mat. Sb. (1964), 525-550.

3. L. Hörmander, Linear partial differential operators, Springer-Verlag, 1976.

4. F. Treves, Linear partial differential equations with constant coefficients, Gordon and Breach, 1966.

MATHEMATICS AND COMPUTER SCIENCE DEPARTMENT, INDIANA UNIVERSITY AT SOUTH BEND, SOUTH BEND, INDIANA 46634

Department of Mathematics, State University of NeW York at Buffalo, BUFFALO, NEW YORK 14214 